INVESTIGACIÓN

\title{
NUEVOS ESCENARIOS PARA EL LIDERAZGO LABORAL Y CONEXIONES RELACIONALES PARA EL DESARROLLO DE EQUIPOS DE TRABAJO HACIA UN COMPETITIVO FUTURO ORGANIZACIONAL*
}

\section{NEW SCENARIOS FOR LABOR LEADERSHIP, AND RELATIONAL CONNECTIONS FOR THE DEVELOPMENT OF WORK TEAMS TOWARDS A COMPETITIVE ORGANIZATIONAL FUTURE}

Recibido: 10 de noviembre de 2017

\author{
Constanza Abadía García ${ }^{\star *}$
}

Evaluado: 04 de diciembre de 2017

Aprobado: 22 de enero de 2017

\section{RESUMEN}

Este artículo presenta un escenario exploratorio que -a partir de los resultados de diferentes informes mundiales y las voces de algunos expertos en áreas académicas, sociales, económicas y del escenario político, asociados al mundo empresarial- expone el advenimiento de nuevos retos para los escenarios de las relaciones laborales, debido a la necesidad de las organizaciones de adaptarse tanto a los cambios de tecnología, como a su incidencia en los estilos de vida e intereses de los consumidores. Dicha emergencia prospecta como crucial, el surgimiento de un liderazgo que

* AArtículo de estudio.

** Psicóloga. Especialista en Evaluación Pedagógica, Especialista en Proyectos Educativos Innovadores, Especialista en Evaluación de la Educación a Distancia en Entornos Virtuales: Perspectivas Innovadoras, Estrategias e Instrumentos. Master of Arts in Education - Higer Education School of Education. Doctorante en Educación y Tecnología de la UNAD-Florida (USA). Miembro del grupo de investigación "Psicología, Desarrollo Emocional y Educación". Actualmente se desempeña como Vicerrectora Académica y de Investigación de la Universidad Nacional Abierta y a Distancia (UNAD). Correo electrónico: Constanza. abadia@unad.edu.co 
movilice los equipos de trabajo para la adquisición y desarrollo de capacidades personales y colectivas, que favorezcan a las empresas u organizaciones en su transición y adaptación en su camino hacia el futuro, aportando la suficiente seguridad y motivación para la elaboración de respuestas innovadoras, ante los problemas que surjan en la cuarta revolución industrial y los nuevos espacios para la competitividad global.

Palabras claves: liderazgo, capital humano, capacidades, disruptivo, consciente

\begin{abstract}
This article presents an exploratory scenario that - based on the results of different world reports and the voices of some experts in academic, social, economic and political settings, associated with the business world - exposes the The advent of new challenges for the scenarios of industrial relations, due to the need of organizations to adapt to both technology changes and their impact on the lifestyles and interests of consumers. This emergency prospects as crucial, the emergence of a leadership that mobilizes the teams of work for the acquisition and development of personal and collective capacities, that favor the companies or organizations in their transition and adaptation in their way Towards the future, providing sufficient security and motivation for the elaboration of innovative responses, to the problems that arise in the fourth industrial revolution and the new spaces for global competitiveness.
\end{abstract}

Key words: Leadership, human capital, capacities, disruptive, conscious. 


\section{INTRODUCCIÓN (LO YA VISTO)}

La excelencia no es producto de las circunstancias. La excelencia es una elección consciente.

Jim Collins ${ }^{1}$

El mundo contemporáneo transita por espacios que vislumbran rápidos cambios en todos los sectores de la sociedad. El más notorio de estos cambios, pese a no ser una primicia para el sector académico y empresarial, es el producido por la revolución progresiva que ha generado el desarrollo de la informática y las demás tecnologías de la información, con una amplia incidencia en los procesos productivos, contribuyendo a la diversificación de la producción en cortos períodos (Jáuregui, Moltó y González, 2004).

Las dinámicas introducidas por el advenimiento de las tecnologías han provocado una nueva perspectiva al revolucionar los escenarios de las relaciones laborales, debido a que coadyuvan a multiplicar la capacidad de acción de las personas, planteando un nuevo modelo laboral que, de manera organizada, impulsa el desplazamiento de un alto número de trabajadores, quienes pueden ser reemplazados por sofisticadas máquinas. En este modelo laboral solo sobrevive el empleado que esté en capacidad de manejar dichas máquinas.

De esta forma, el camino que derivó en este modelo parece no tener regreso, pues el mercado también se adecuó a la producción originada con el favor de estas tecnologías, entre las que se encuentra la digital. Sumado a lo anterior, las necesidades de marketing empresarial, la oferta de productos y los servicios se ven abocados a una permanente y rápida adaptación, ante la emergente demanda de movilidad que el mercado plantea a las empresas, la cual beneficiará en mayor medida a aquellas que identificaron los cambios y asumieron una rápida transformación y adaptación en sus dinámicas de producción, promoción y, no menos importante, en las formas en que presenta el liderazgo organizacional y las relaciones de este con los equipos de personas, a quienes necesita potenciar y movilizar para el nuevo desarrollo empresarial.

\section{LO QUE VEMOS Y LO QUE EMPEZAMOS A VER}

No es infundado que el reporte que surgió de la encuesta anual de Deloitte', titulado Reescribiendo las reglas para la era digital:

1 Matemático, docente e investigador de la Escuela de Negocios de la Universidad de Stanford, consultor de negocios, escritor y conferenciante sobre temas de gestión empresarial.

2 Deloitte Touche Tohmatsu Limited (Deloitte), por volumen de facturación, es la firma privada número uno de servicios profesionales del mundo (Londres, septiembre 14 de 2017: US \$ 38,8 mil millones para el año fiscal finalizado el 31 de mayo de 2017), (Deloitte, 2017). 
Reporte de tendencias globales en capital humano 2017, plantee transversalmente a "lo digital" como uno de los resultados más notorios -sumado a lo económico y lo demográfico-, y lo exponga como uno de los desafíos para los líderes empresariales y de recursos humanos, debido al acelerado cambio que produce en las reglas sobre como organizan, reclutan, desarrollan, gestionan y comprometen a la fuerza laboral del siglo $\mathrm{XXI}^{3}$ (Deloitte, 2017). Esto último también es un llamado a las instituciones de educación superior, para que formen a nuevos profesionales con altas competencias digitales, junto con otras de tipo polivalente que se requieren para el acceso a nuevos escenarios del campo laboral.

En el mismo sentido, Jim Yong Kim, director del Banco Mundial, declaró ${ }^{4}$ que "la inteligencia artificial va a eliminar entre el $50 \%$ y el $65 \%$ de todos los trabajos existentes en los países en vías de desarrollo [...]" y añadió, "[...] esta dinámica vibrante va a generar nuevos trabajos porque nuestra tarea no es tratar de preservar los empleos antiguos, sino crear nuevos que van a necesitar nuevas capacidades". En consecuencia hizo un llamado a los gobiernos a promover políticas que impulsen la aparición de nuevos ecosistemas de emprendedores, exhortando a "que los esfuerzos se vuelquen al desarrollo de una educación de mayor calidad, capaz de fomentar el pensamiento crítico y la solución de problemas", consecuente con su afirmación acerca de que "la innovación jugará un importante rol en el escenario económico mundial".

Es evidente que no existen precedentes históricos sobre el acelerado ritmo en los avances que produce la tecnología actual, como es el caso del surgimiento de la inteligencia artificial, las plataformas móviles, los sensores, los sistemas de registro de transacciones, y los sistemas de colaboración social, los cuales, están revolucionando los modos y formas en que trabaja y se comunica la sociedad contemporánea, cambiando aun la manera como se observan los hechos concretos de lo real o sus manifestaciones, y entonces, también en el cómo se advierten las posibilidades de realidad que de ello surgen.

A la par con las nuevas percepciones que adquieren las personas, y aunque el incremento de la tecnología es acelerado y las de "la

3 Deloitte (2017). Reescribiendo las reglas para la era digital: Reporte de Tendencias Globales en Capital Humano 2017.

4 "Invirtiendo en los empleos del futuro", panel realizado el viernes 18 de agosto de 2017 en el Ministerio de Ciencia y Tecnología de Buenos Aires, Argentina. 
persona" incrementa lentamente y de manera lineal, también está su notoria habilidad para adaptarse rápidamente a las innovaciones (Friedman, 2016), marcando una obligada ruta a las industrias para la incorporación de servicios digitales que les permitan atraer la atención de los consumidores.

El Global Startup Ecosystem Report 2017, expone que la era de la información está floreciendo.

La tecnología está generando riqueza e innovación a un ritmo exponencial, pero solo un puñado de lugares en el mundo captura la mayor parte de esa creación de valor. En todas partes, las regiones y las personas se están quedando atrás. Sin acciones inmediatas y agresivas para desarrollar ecosistemas de inicio más fuertes, esta divergencia continuará y más lugares se perderán el crecimiento tecnológico y el dinamismo. Si bien la tecnología siempre crea ganadores y perdedores, hoy la brecha entre ellos se está ampliando a un ritmo rápido. (Global Entrepreneurship Network, 2017, p. 9).

El mismo informe plantea el surgimiento de tres desafíos económicos, que se encuentran en las brechas que producen: la divergencia entre las regiones geográficas impulsadas por la tecnología, la concentración de nuevas empresas y su valor, e igualmente, la creciente desigualdad entre los diferentes tipos de trabajadores debido a esa concentración. Aunque estas brechas no parecen ser nuevas, el informe advierte que la expansión del sector tecnológico -y su infiltración en otros sectores- las está exacerbando, y asevera que "solo los ecosistemas de inicio fuertes pueden garantizar una participación y beneficios más amplios, y todas las regiones deben asumir urgentemente esta tarea" (2017, p. 10).

Para mencionar un ejemplo, se puede aprovechar el que nace de una de las respuestas que Christine Lagarde, directora gerente del Fondo Monetario Internacional, dio a The Wall Streeet Journal cuando se le preguntó sobre las monedas digitales y la tecnología Blockchain ${ }^{5}$, como uno de los temas que afectan al mundo económico en el que vivimos, ante lo cual manifestó lo siguiente:

En última instancia, creo que va a estar en el interés de las instituciones financieras el adoptar esas tecnologías digitales, porque,

5 Blockchain es la plataforma de software líder en el mundo para activos digitales, resultante de la combinación de tecnologías probadas y aplicadas de una nueva manera: Internet, criptografía de clave privada y un protocolo que gobierna la incentivación. Representa una innovación en el registro y la distribución de información que elimina la necesidad de que una parte confiable facilite las relaciones digitales. 
como cualquier actor económico en ese campo, quieren llegar a nuevos mercados. Ellos quieren no sólo mantener su base de mercado, sino expandirlo. Y la manera de ampliarlo es llegando a nuevos territorios, [por lo que] estaría muy sorprendida si en 5 años las instituciones financieras existentes no adoptasen estas herramientas. (Lagarde, entrevista, 3 de octubre de 2016).

A pesar de que, por profusión mediática, la tecnología Blockchain o cadena de bloques es generalmente asociada al mencionado producto, el ejemplo se orienta hacia lo que expone Marc Andreessen": "La consecuencia práctica es por primera vez, una forma para que un usuario de Internet transfiera una propiedad digital única a otro usuario de Internet, de modo que la transferencia garantizada sea segura, todos saben que la transferencia ha tenido lugar, y nadie puede desafiar la legitimidad de la transferencia. Las consecuencias de este avance son difíciles de exagerar" (Coindesk, en línea).
A partir del aporte de la tecnología Blockchain, han surgido innovaciones como la que registra el MIT Technology Review?: "la empresa de transporte Maersk ha anunciado sobre su colaboración con International Business Machines (IBM), para emplear la cadena de bloques para rastrear los envíos marítimos" (Condliffe, 2017). Pero lo relevante no es el rastreo de sus contenedores sino sus contenidos, que ahora y para ejemplificar pasarían de necesitar de 30 personas realizando papeleos repartidos entre 200 interacciones o más (del este de África hasta Europa), a solo dos interacciones: cliente-proveedor-cliente, reduciendo la necesidad de mano de obra a uno o dos trabajadores. Actualmente IBM está colaborando con 400 organizaciones distintas para aplicar la tecnología a diferentes situaciones.

El panorama presenta como preponderante la necesidad de las organizaciones de adaptarse tanto a los cambios de tecnología, como

6 Forbes Media LLC (2017). Perfil: Uno de los principales socios de la sociedad de capital de riesgo Andreessen Horowitz, con Instagram y Oculus como inversiones exitosas; cofundador del navegador web Netscape; su mayor puntuación fue alcanzada como un inversor de semilla en Facebook. Anecdóticamente se le adjudica el que bajo su coaching, Marc Zukerberg empezó a comportarse como un auténtico líder. Ver: https://www.forbes.com/profile/marc-andreessen/. (Consultado el 23 de septiembre de 2017).

7 Revista publicada por Technology Review Inc., compañía independiente de medios de comunicación propiedad del Instituto Tecnológico de Massachusetts (MIT). 
a los estilos de vida e intereses de los consumidores, lo cual determina un cambio del liderazgo ante el paradigma que se desarrolló en la primera etapa industrial sobre las prácticas de negocio de planeación corporativa, estructura organizacional, diseño de puestos, establecimiento de objetivos y gestión; más cuando el nuevo ámbito al que se enfrenta ha sido denominado la cuarta revolución industrial (Schwab, 2016).

En medio de la magnitud de avances tecnológicos y ante la incertidumbre que trae para cierto tipo de trabajadores con mano de obra no calificada en las empresas del futuro inmediato y venidero, irrumpe un nuevo sistema de pensamiento que se pone de manifiesto en distintos campos del conocimiento, como resultado del nuevo entramado económico, político y social que acontece, y a partir del cual, el mundo moderno define sus nuevos discursos y prácticas. La emergencia de nuevos problemas y un conjunto de respuestas que se necesitan elaborar en función de las nuevas condiciones históricas emergentes, invita a concebir nuevas formas y modos de un tipo de liderazgo que facilite el conectar las diferencias entre un mundo que se abandona y otro que emerge.

\section{NO ES SUFICIENTE EL LIDERAZGO QUE HEMOS VISTO}

¿Quién es más humilde? ¿El científico que mira el universo con una mente abierta y acepta todo lo que tiene que enseñarnos, o alguien que dice que todo lo dicho en un libro debe considerarse la verdad literal sin importar la falibilidad de todos los seres humanos involucrados?

Carl Sagan ${ }^{8}$

Bajo este panorama, no es extraño que para pasar de la producción en serie y estandarizada a la producción diversificada y, en últimas, para alcanzar una mayor productividad y competitividad, se necesite de la incorporación de dichas tecnologías a las industrias y a las empresas de servicios; sin obviar que para alcanzar un adecuado desarrollo es vital el surgimiento y la adaptación a nuevas formas de gestión del trabajo, pues como expone el reporte Deloitte: "Las organizaciones enfrentan un cambio radical en el contexto de la fuerza laboral, el lugar de trabajo y el mundo laboral" (Deloitte, 2017, p. 3).

Como se ha expuesto, para lograr dicho cometido no es suficiente con el simple ingreso de nuevos desarrollos tecnológicos al mundo empresarial y de las organizaciones, también

8 Considerado el astrónomo, astrofísico, cosmólogo, escritor y divulgador científico más importante de los Estados Unidos en los años 80 y a principios de los 90. 
es fundamental contar con talento humano y equipos de trabajo que puedan responder ante tales expectativas, además de un tipo de liderazgo que proporcione la suficiente seguridad y motivación para alcanzar las metas propuestas. Esto incluye el desarrollo de nuevas tecnologías, en este caso, para el manejo y direccionamiento del capital humano. Se evidencia así la necesaria inclusión de otros procedimientos, de una diferente localización para el sujeto, de una nueva configuración de directrices y prácticas, que mutan y demandan de la polivalencia laboral en función de un reacomodamiento en las relaciones de poder.

Entre las diez tendencias que arroja el reporte Deloitte, y para el interés del tema que aborda el presente artículo, se destacan la "carrera y aprendizaje: en tiempo real, todo el tiempo" y el "liderazgo disruptivo: desafiando los límites". Esto se fundamenta en que las organizaciones, al mismo tiempo que incorporan tecnologías de punta, necesitan experiencias de aprendizaje continuo, para que en los propios términos de los colaboradores se construyan y desarrollen habilidades para el efecto, de forma rápida y sencilla (Deloitte, 2015); así mismo, las organizaciones necesitan de una transformación que, simultáneamente con el modelo organizacional digital emergente, adopte una concepción del liderazgo con condiciones ágiles y polivalentes, que permitan la prosperidad en una red que cambia rápidamente.

Al igual que la comunicación debe ser un instrumento permanente de acercamiento entre personas y de éstas con sus respectivos líderes, ella necesita que los líderes posean una forma de pensar que en el proceso de cambio organizacional permanente, visualice alternativas que les permitan interrumpir el cauce tradicional de las cosas, para guiar a sus equipos a alcanzar logros consistentes y predecibles; es decir, que adquiera y desarrolle "pensamiento disruptivo" para facilitar que la comunicación sea más directa y transparente, permitiendo que se comprenda y se pueda aportar a los pensamientos que los líderes efectivos y reflexivos tienen (Hoque, 2015), como medio para conformar equipos de trabajo eficaces con altos niveles de excelencia y competitividad.

El profesor Clayton Christensen ${ }^{9}$, en su libro El dilema de los innovadores, acuñó el término "innovación disruptiva", para describir un tipo de innovación en la que un producto o servicio se va desarrollando mediante su introducción sencilla

9 Empresario y profesor de la Harvard Business School, clasificado en el puesto 1 de los Thinkers50, el ranking mundial de líderes empresariales creado por Des Dearlove y Stuart Crainer. Es el arquitecto y la autoridad más importante del mundo en la innovación disruptiva. Fue nombrado el Pensador de Gestión de Negocios más influyente del mundo en 2011 y 2013. 
a la parte más baja de un mercado, hasta que, progresivamente y sin descanso, se mueve hacia la parte más alta de dicho mercado, desplazando finalmente a los competidores establecidos (Christensen, 1999).

Según Christensen, en la gestión y dirección es en donde se produce una de las diferencias más notorias entre las empresas grandes y establecidas, frente a los que están interrumpiendo sus mercados desde la parte inferior de la curva de crecimiento del sector. Por lo tanto, con seguridad se puede manifestar que el liderazgo tiene una de las mayores responsabilidades para asegurar la supervivencia de la organización, la rentabilidad, la sostenibilidad y la pertinencia a largo plazo, en tiempos de aceleración de la innovación y el cambio. Este tipo de liderazgo, al que se ha denominado liderazgo disruptivo, implica que los líderes sean curiosos, de mente abierta, con capacidad para guiar a sus equipos a través de la incertidumbre, y que salgan del confort de las normas establecidas.

Los líderes disruptivos son personas curiosas del mundo que les rodea, tanto en lo inmediato como en lo distante, permitiendo que su rol evolucione pasando de decir qué hacer, a plantear y dirigir la resolución de las preguntas más difíciles que cualquiera puede plantear. Solo con la mente abierta pueden ser interlocutores eficaces. En palabras de Christensen:
En primer lugar, porque en tiempos de exponencial de la información y la creación de conocimiento, es probable que tengan las respuestas a no más de $0,01 \%$ de todas las preguntas. Y, en segundo lugar, porque esas preguntas podrían crear respuestas que están en conflicto con la perspectiva de los líderes, sin embargo, que llevan dentro, el poder de crear mercados completamente nuevos o valor de oferta a niveles nunca antes vistos (Christensen, 1999, p. 297).

Los líderes disruptivos se sienten cómodos en un ambiente de pensamiento perturbador, pues ante las necesidades de innovar frente a los rápido cambios, ven oportunidades en la incertidumbre, lo que les permite convertirse en el germen que organizacionalmente aporta inspiración, ante presiones fuertes para el o los equipos que lidera. Sumado a estas características, perciben las normas como estandarizaciones que limitan las posibilidades de cambio e innovación al estar centradas en la sola eficiencia; en cambio, los líderes disruptivos alientan el cuestionamiento y la movilidad de pensamiento como escenario adecuado para la innovación, siendo capacidades que deberían darle cabida en cualquier empresa u organización que tienda a mayores niveles de desarrollo.

Lo anterior muestra la calidad que adquiere un líder disruptivo, en la medida que su curiosidad, apertura mental, capacidad de guía ante 
la incertidumbre y de visión para innovar ante los impedimentos de lo preestablecido, se convierten en valores agregados que responden a la oferta de un mercado laboral que está tras la búsqueda de talentos que aporten a nuevas formas para la potenciación de las capacidades de las organizaciones, las cuales procuran el reclutamiento de perfiles que contribuyan con trabajo creativo mediante sus conocimientos y destrezas aplicadas.

\section{EL LUGAR DE LO PROSPECTIVO EN EL LIDERAZGO}

Desde la comprensión del Instituto de Prospectiva Estratégica de España, la prospectiva

es una disciplina con visión global, sistémica, dinámica y abierta que explica los posibles futuros, no sólo por los datos del pasado sino fundamentalmente teniendo en cuenta las evoluciones futuras de las variables (cuantitativas y sobre todo cualitativas) así como los comportamientos de los actores implicados, de manera que reduce la incertidumbre, ilumina la acción presente y aporta mecanismos que conducen al futuro aceptable, conveniente o deseado. (Instituto de Prospectiva Estratégica, en línea).

Lo que le acredita un tipo de valor en tanto es "entendida en una forma contemporánea, como una disciplina que facilita la anticipación y construcción de futuros de la sociedad" (Medina y Mojica, 2008, p. 193).

Por otra parte, en el inicio del prólogo del libro La empresa consciente de Fredy Kofman ${ }^{10}$, Ken Wilber ${ }^{11}$ anota que la definición aburrida y prosaica de empresa es: "Intento o designio de hacer una cosa. Casa o sociedad mercantil o industrial fundada para llevar a cabo construcciones, negocios o proyectos de importancia. Obra o designio llevado a efecto, en especial cuando en él intervienen varias personas" (Kofman, 2012, p. 7), y lo contrasta con el concepto de "ser consciente", que significa

percibir el mundo interior y exterior. Que siente, piensa, quiere y obra con conocimiento de lo que hace. Con pleno uso de los sentidos y facultades. Por lo tanto, la expresión "empresa consciente"

10 Escritor, filósofo y coach argentino. Director del Conscious Business Center de la Universidad Francisco Marroquín, y presidente del Consejo Académico de la consultora internacional Axialent.

11 Ken Wilber (Oklahoma, 1949) es uno de los más influyentes pensadores de Estados Unidos, y una de las más intrigantes figuras que ha dado la intelectualidad de dicho país en los últimos años. Autor prolífico, es considerado el principal estudioso de la conciencia, la psicología transpersonal y el enfoque integral. Ver http://editorialkairos.com/autores/wilber-ken. Consultado el 29 de septiembre de 2017. 
podría definir la capacidad de intentar hacer una cosa, de llevar a cabo construcciones, negocios o proyectos de importancia, en especial cuando intervienen varias personas, de una manera consciente, con pleno uso de los sentidos y facultades. Lo cual, por supuesto, implica que muchas personas no lo hacen de esa manera (Kofman, 2012, p. 7).

Al observar y analizar la relación entre lo prospectivo y el ser consciente, aparece un tipo de diálogo entre los conceptos, que proyecta una visión diferente del mundo, no anquilosada por lo que se vio o vivió, sino antes bien por una postura visionaria de futuro, como plataforma para la incubación del pensamiento disruptivo, hacia la generación de un tipo de actuación en el escenario empresarial y de las organizaciones, al que en palabras de Fredy Kofman y Robert Dilts $^{12}$, se denomina: el liderazgo consciente.

El punto para nuestro escenario, podríamos visualizarlo como afín con algunas de las posturas que contiene el documento: "Una nueva agenda de capacidades para Europa - Trabajar juntos para reforzar el capital humano, la empleabilidad y la competitividad" (Comisión Europea, 2016), en

el que se declara como

sorprendente la cantidad de carencias y desfases que existen en el terreno de las capacidades $^{13}$. Muchas personas ocupan puestos de trabajo que no se corresponden con su talento [...] los empleadores tienen problemas para encontrar a personas con las capacidades que necesitan para crecer e innovar. Los proveedores de educación, por un lado, y los empleadores y los estudiantes, por otro, tienen distintas percepciones sobre la adecuada preparación de los titulados para el mercado laboral. (Comisión Europea, 2016, p. 2).

Así entendido, la adquisición y el desarrollo de capacidades se convierten en cruciales para el funcionamiento y la modernización de los mercados laborales, con el fin de ofrecer nuevas formas de flexibilidad y seguridad para los demandantes de empleo, los trabajadores y los empleadores, más cuando la transformación digital de la economía está reconfigurando la manera de vivir y hacer negocios en nuestras sociedades. Han aparecido nuevas

12 Reconocido internacionalmente como uno de los primeros desarrolladores, formadores y profesionales de la Programación Neuro-Lingüística.

13 El término «capacidades" se utiliza en un sentido amplio, para hacer referencia a aquello que una persona sabe, comprende y es capaz de hacer. (p. 2). 
modalidades de trabajo que afectan a los tipos de capacidades que se precisan (Comisión Europea, 2016).

Las personas necesitan un amplio conjunto de capacidades para desarrollar plenamente su potencial en el trabajo y en la sociedad. En el mejor de los casos, disponer de capacidades de alto nivel facilita que las personas puedan adaptarse a los cambios imprevistos, además de las implicaciones que conlleva sobre la creación y adaptación de los modelos empresariales y de los perfiles de los puestos de trabajo. Sin embargo, lo anterior no se da de forma espontánea y mucho menos por una exposición prolongada a la necesidad, hasta que surja la solución.

La elaboración de respuestas a los problemas que surjan, es decir, la construcción de soluciones determinará el grado de éxito o fracaso al que llegue una empresa u organización. Pero la solución no está en la tecnología, sino en su manipulación a favor de lo que se proyecte. Es en este escenario en el que las personas y el liderazgo que los guíe se convierte en trascendental, pues solo en la medida en que el liderazgo movilice el proceso mediante el cual se determina un objetivo que deberán lograr otras personas y logre motivarlas a perseguir la concreción de ese objetivo con eficacia y compromiso pleno, se podrá transformar el potencial individual en desempeño colectivo, para alcanzar una visión compartida.

\section{ENTONCES... ¿QUÉ TIPO DE LIDERAZGO SE NECESITA?}

Sólo un guerrero es capaz de soportar el camino del conocimiento. Un guerrero no puede quejarse o lamentar nada. Su vida es un permanente desafío y los desafíos no son buenos ni malos. Los desafíos son simplemente desafíos. La diferencia básica entre un hombre ordinario y un guerrero es que el guerrero toma todo como un desafío, mientras que el hombre ordinario toma todo como una bendición o una maldición.

Juan Matus ${ }^{14}$

Los términos leading (guiar) y leadership (liderazgo) provienen del viejo vocablo inglés lithan, que significa ir. Según el Diccionario MerriamWebster, liderazgo significa "guiar sobre un camino, especialmente al ir por delante". Entonces el liderazgo tiene que ver frecuentemente con "ir primero" e influir en los demás, tanto a través de las acciones como de las palabras. En consecuencia, el liderazgo se manifiesta en la "capacidad para expresar una visión, influenciar a otros a lograr resultados, alentar la cooperación grupal, y ser un ejemplo" (Webster, 1828, en línea).

14 Chamán Yaqui, citado en el recuento etnográfico de la obra del antropólogo peruano Carlos Cataneda, Las enseñanzas de Don Juan y Viaje a Ixtlán. 
Fredy Kofman plantea que cuanto mejor conozcamos el mundo interior y el exterior, es decir, cuanto mejor los comprendamos, tanto mejor podremos recorrerlos. De esta manera acoge el liderazgo consciente como una postura de vida, que trata acerca "del ser, más que del conocer, "de la emoción, más que de la cognición", "del espíritu, más que de la materia"; sin demeritar su correlación, pues tampoco encuentra contradicción entre la riqueza material y la espiritual. Este tipo de liderazgo implica construir soluciones desde un estado de presencia centrada, que recupere las posibilidades que brindan las inteligencias múltiples ${ }^{15}$ y el vivir los valores más altos en el servicio para el alcance de un propósito mayor, lo que demanda su autenticidad, responsabilidad, propositividad y, no menos importante, su inteligencia emocional.
Debido a la incertidumbre que siempre muestran las posibilidades de futuro, este liderazgo debe ser capaz de mantener una sólida actitud mental sobre lo que se piensa, para movilizar las acciones que se necesitan para enfrentar los retos, y, en síntesis, alcanzar los resultados esperados. Esto se interpreta como un liderazgo con capacidad de formular y comunicar una visión clara y significativa sobre el futuro, siendo entonces, un liderazgo que mediante un análisis prospectivo identifica y actúa sobre escenarios posibles, establecidos mediante el análisis de un presente reflexivo que tiene en cuenta las lecciones aprendidas de la experiencia; y a su vez, le permite, a través del ejemplo, servir como inspiración para sus colaboradores, lo cual solo encontrará al desarrollar un adecuado equilibrio entre el interés personal y el bien común, en sí mismo y en otros, respetando e integrando en su actuar el surgimiento de múltiples perspectivas para la concepción de innovaciones en los posibles escenarios de futuro ${ }^{16}$.

15 La teoría de las inteligencias múltiples es un modelo de concepción de la mente propuesto en 1983 por el psicólogo estadounidense Howard Gardner, profesor de la Universidad de Harvard, para quien la inteligencia no es un conjunto unitario que agrupe diferentes capacidades específicas, sino una red de conjuntos autónomos relacionados entre sí. (Blanes, en línea).

16 Kees Van der Heijden afirma que "Ios escenarios deben ser la representación de una perspectiva innovadora, nueva y original del futuro. Entonces, el escenario no es un fin en sí mismo, pues no tiene sentido si no es útil para la elección de las opciones estratégicas. Interesa en la medida que interviene en los resultados y en las consecuencias que entraña para las propias opciones estratégicas. Los escenarios son un medio eficaz para tomar decisiones y no para adivinar lo que podría acontecer" (citado por Mojica, 2005, p. 50). 
Jim Collins y Jerry I. Porras ${ }^{17}$, citados por Kofman, plantean que de negocios, "maximizar la riqueza del accionista" o la "maximización de ganancias" no han sido los objetivos predominantes [...] de las compañías excepcionales [...]. Por supuesto, se orientan a obtener ganancias, pero están igualmente guiadas por [...] valores fundamentales y un sentido de su misión que excede el fin de lucro. Incluso, paradójicamente, las compañías visionarias ganan más dinero que aquellas que, en comparación, están puramente orientadas a obtener ganancias. (Kofman, 2012, p. 374) contrariamente a lo que enseñan las escuelas

- Capacidades asociadas al dominio de una actitud mental óptima

- La creación de un futuro claro y atrayente para sus colaboradores y otros equipos de trabajo

- Establecer una ruta crítica hacia el estado deseado que traza la visión y objetivos organizacionales

- Alcanzar una alineación personal e interna con las metas de la organización y las de sus miembros

- Desarrollar alianzas poderosas, internas y externas a la organización

Teniendo en cuenta lo expuesto por Collins y Porras, y ante los cambios que ha traído al avance tecnológico en los ámbitos económico, político y social, se vislumbra un liderazgo que podría guiar a las empresas u organizaciones a la transición y adaptación. Lo anterior involucra una adecuada dinámicas del rumbo propuesto.
- Transformación efectiva de obstáculos

\section{- Propiciar un sentido práctico que permita im- plementar correcciones para ajustarse a las} gestión del recurso humano, con el fin de que se

puedan dar las siguientes condiciones:

17 Basándose en un proyecto de investigación de seis años en la Escuela de Graduados de la Universidad de Negocios de Stanford, Collins y Porras tomaron dieciocho empresas verdaderamente excepcionales y de larga duración - que tienen una edad media de casi un centenar de años y han superado al mercado de valores en general por una el factor de quince años desde 1926- y estudiado cada empresa en comparación directa con uno de sus principales competidores. Examinaron las empresas desde sus inicios hasta la actualidad - como puesta en marcha, ya que las empresas medianas y las grandes corporaciones. En todo momento, los autores preguntaron: "¿Qué hace que las empresas verdaderamente excepcionales diferentes de otras compañías?". Consultado en: https://www.harpercollins.com/9780062119087/built-to-last 
Además de lo anterior, se debe tener presente que el liderazgo es ejercido por personas y su vivencia se materializa a partir de la calidad de las conexiones relacionales que se construyan con otros. En ese sentido, las emociones son parte esencial del desarrollo de las capacidades, pues una emoción es una condición que afecta al organismo en su totalidad e influye en la efectividad con que una persona interactúa con su entorno; incluso representa un cambio en el estado interno de la persona, en su forma de actuar y, lo más importante, en la manera de influir sobre aquellos que lo rodean.

El liderazgo que se ha descrito a lo largo de este artículo también necesita de la inteligencia emocional. Daniel Goleman (citado por Fernández-Berrocal y Extremera, 2009, p. 90), redefinió el concepto de liderazgo, de dos formas: la primera formulación lo asocia con el "carácter" de la persona (Goleman, 1995, 28); la segunda lo concibe como un conjunto de competencias socio-emocionales relacionadas con el éxito en el ámbito laboral (Goleman, 1998), lo cual podría describirse como un tipo de habilidad que facilita identificar y monitorear las emociones personales y de los otros, con el fin de diferenciar entre ambas y emplear la información generada para guiar el proceso cognitivo y la actuación. Sin ella, el líder solo estaría en una condición nominal.

\section{A MODO DE CONCLUSIONES}

Todas las ideas y conceptos esbozados en este artículo, proponen una mirada que busca la reflexión sobre lo aprehendido, comprendiendo que el competir por el futuro está íntimamente ligado a imaginarlo, para después crearlo. En dicho sentido, sería paradójico que al menos en el diseño al nivel de pensamiento organizacional, solo algunas empresas logren un futuro menos incierto, y las que no, dejen de ser líderes para convertirse en seguidoras de las que enfrenten de mejor manera el futuro, pues como no hay una única empresa, tampoco se prospecta un único tipo futuro para todas ellas. Crear un futuro propio implica el reto de construir un mapa consecuente con el camino personal, lo cual es más gratificante que seguir el camino trazado por otros.

Las empresas y organizaciones del presente-futuro demandarán un tipo de liderazgo no solo personal -asociado a sus trabajadoressino también organizacional, con el que antiguos y nuevos empleados logren desarrollar más y mejores capacidades, de cara a los retos que se aproximan. De esta forma las empresas podrán alcanzar una adecuada evolución y ajuste ante el panorama que encuentran, y potencialmente acierten en la vivencia de un liderazgo que les provea de personas con espíritu de lucha, que como guerreros sepan sortear las dificultades, acogiendo cada desafío que se presente como 
un nuevo paso para crear y dominar las oportunidades que van surgiendo, y de este modo, definir un nuevo espacio competitivo en su camino hacia el futuro organizacional.

\section{REFERENCIAS}

Blanes, A. (s.f.). La teoría de las inteligencias múltiples. Descripción breve. ¿Qué es, qué describe y qué tipos de inteligencia existen? [en línea]. Buenos Aires, Argentina: Universidad de Buenos Aires, disponible en http://bioinformatica.uab.cat/base/documents/genetica_gen/ portfolio/La\%20teor\%C3\%ADa\%20de\%20 las $\% 20$ Inteligencias $\% 20 \mathrm{~m} \% \mathrm{C} 3 \%$ BAltiples $\% 20$ 2016_5_25P23_3_27.pdf

Cataneda, C. (1972). Viaje a Ixtlán. Ciudad de México, D. F., México: versión originar en inglés publicado por: Simon \& Schuster. Versión en español: Fondo de Cultura Económica.

Catañeda, C. (1968). Las enseñanzas de Don Juan: Una forma Yaqui de conocimiento. Estados Unidos de Norte América: Universidad de California.

Coindesk (s. f.) What is Blockchain Technology? [en línea]. Disponible en https://www.coindesk.com/ information/what-is-blockchain-technology/

Condliffe, J. (3 de marzo de 2017). La expansión de «blockchain` por toda la industria llega al transporte de mercancías [en línea]. Woods, T. (Trad.). En MIT Technology Review, Disponible en https://www.technologyreview.es/s/6862/ la-expansion-de-blockchain-por-toda-la-industria-Ilega-al-transporte-de-mercancias. [Consultado el 28 de septiembre de 2017].

Comisión Europea (junio 10 de 2017). Comunicación de la Comisión al Parlamento Europeo, al Consejo, al Comité Económico y Social Europeo y al Comité de las Regiones. [en línea]. Disponible en https://eur-lex.europa.eu/legal-content/ES/ ALL/?uri=CELEX\%3A52014DC0015. [Consultado el 22 de septiembre de 2017].

Deloitte. (2017). Reescribiendo las reglas para la era digital: Tendencias globales en capital humano 2017 [en línea]. Disponible en https://www2.deloitte.com/content/dam/ Deloitte/cr/Documents/human-capital/estudios/170228-DUP_Global-Tendencias-CapitalHumano_2017.pdf. [Consultado el 25 de septiembre de 2017].

Deloitte. (2015). Deloitte survey: Americans look at their smartphones in the aggregate more than 8 billion times daily [en línea]. En PRNewswire. Disponible en http://www.prnewswire.com/news-releases/ deloitte-survey-americans-look-at-their-smartphones-in-the-aggregate-more-than-8-biIlion-times-daily-300190192.html. [Consultado el 25 de septiembre de 2017]. 
Fernández-Berrocal, P. y Extremera, N. (2009). La inteligencia emocional y el estudio de la felicidad. Concepto de inteligencia emocional. En Revista Interuniversitaria de Formación del Profesorado, 66. p. 90. [en línea]. Disponible en http://www. redalyc.org/html/274/27419066006/

Forbes Media LLC (2017). Profile: Andreessen Horowitz [en línea]. Disponible en https:// www.forbes.com/profile/marc-andreessen/. [Consultado el 23 de septiembre de 2017].

Friedman, T. (2016). Thank you for being late: An Optimist's Guide to Thriving in the Age of Accelerations.Publicado por Farrar, Straus y Giroux. New York, Estados Unidos de Norte América.

Global Entrepreneurship Network (GEN) (2017). Global Startup Ecosystem Report 2017, The Urgency of Ecosystem Investments. Estados Unidos de Norte América, Oakland: Startup Genome.

Goleman, D. (1995). Emotional Intelligence. New York: Bantam Books. (Trad. Cast. Kairós, 1996). New York, United States of North America.

Hoque, F, (2015). Survive to Thrive - 27 Practices of Resilient Entrepreneurs, Innovators and Leaders. Estados Unidos de Norte América, Carlsbad (California): Motivational Press Incorporated.
Instituto de Prospectiva Estratégica (s.f.). Instituto de Prospectiva Estratégica [en línea]. Disponible en http://www.prospecti.es/ipeframe.htm. [Consultado el 21 de septiembre de 2017].

Jáuregui, R., Moltó, J. y González de Lena, F. (2004). Un futuro para el trabajo en la nueva sociedad laboral. Valencia, España: Editorial Tirant Lo Blanch.

Kofman, F. (2012). "La empresa consciente". Cómo construir valor a través de valores. Publicado por Grupo Editorial Penguin Random House. Nueva York, Estados Unidos de Norte América.

Medina, J. y Mojica, F. (2008). La prospectiva en Colombia: Antecedentes, lecciones y desafíos. En Prospectiva na America Latina. Evolução e Desafios. Brasil: Canal 6 Projetos Editoriais.

Merriam-Webster Dictionary (1828). Búsqueda del término "lead" [en línea]. Springfield, Massachusetts, Estados Unidos de Norte América. Disponible en http://www.merriam-webster.com/dictionary/ lead [Consultado el 26 de septiembre de 2017].

Merriam-Webster Dictionary (1828). Búsqueda del término "leader". [en línea]. Springfield, Massachusetts, Estados Unidos de Norte América. Disponible en http://www.merriam-webster.com/ dictionary/leader [Consultado el 26 de septiembre de 2017] 
Ministerio de Ciencia y Tecnología. "Invirtiendo en los empleos del futuro". Panel realizado el viernes 18 de agosto de 2017, en el Ministerio de Ciencia y Tecnología de Buenos Aires, Argentina.
World Economic Forum. Disponible en https:// www.weforum.org/agenda/2016/01/the-fourth-industrial-revolution-what-it-means-andhow-to-respond/ [Consultado el 19 de septiembre de 2017]

Mojica, F. (2005). La construcción del futuro. Concepto y modelo de prospectiva estratégica, territorial y tecnológica. Bogotá, D.C., Colombia: Convenio Andrés Bello. Universidad Externado de Colombia.

Sudeep, R. (3 de octubre de 2016). Entrevista a Christine Lagarde, directora gerente del Fondo Monetario Internacional [en línea]. Disponible en http:// www.wsj.com/video/christine-lagarde-automation-could-open-up-new-jobs/A9EC8DCC-7577-

Schwab, K. (2016). The Fourth Industrial Revolution: what it means, how to respond [en línea]. En 4755-AF63-1EE701413028.html. [Consultado el 26 de septiembre de 2017] 